\title{
Key Point Recognition Method of Bacterial Image in Water Based on Complex Network
}

\author{
Zou Qingyu \\ College of Electrical and Information Engineering \\ Beihua University \\ Jilin, China \\ zouqingyu2002@126.com
}

\author{
Wang Tao \\ College of Electrical and Information Engineering \\ Beihua University \\ Jilin, China \\ wt1997123@foxmail.com
}

\author{
Qian Yun* \\ College of Electrical and Information Engineering \\ Beihua University \\ Jilin, China \\ 924732859@qq.com
}

\begin{abstract}
Water is the material basis of human life. In recent years, water pollution has received more and more attention. The degree of water contamination by bacteria can make a correct assessment by detecting microbes in water. In this paper, an image recognition technique based on complex network is proposed, which is used to detect the key pixels of bacteria in water. The experimental results show that this method can effectively identify the key pixels of bacterial images in water.
\end{abstract}

Keywords-bacterial image; key point recognition; complex network; water

\section{INTRODUCTION}

With the development of computer technology, computer image processing and analysis technology plays an increasingly important role in the sewage treatment process. Digital image processing and pattern recognition technology has been widely used in the field of sewage treatment. Bacterial image processing and analysis with a computer can be more accurate than the visual analysis method. The computer image processing and analysis of sewage bacterial images mainly include image acquisition, image preprocessing, key pixel recognition, and feature analysis, output and so on. The quality of the key point recognition results directly affects the quality of image analysis, recognition and interpretation, which is of great significance, which is also the difficulty and the most important step in the automatic interpretation and analysis of bacterial images. Therefore, many scholars at home and abroad from the last century began to study this area. After more than 40 years of research, thousands of various types of key node recognition algorithms have been proposed. Nevertheless, the study of experts and scholars in this area has not stopped, but has been actively engaged in trying to find more effective and more common algorithm. Many of the existing technologies

This work is supported mostly by the Key Education Research Project of Beihua university, partially by the Jilin Province Science and Technology Development Project(Grant NO. 20170520057JH), partially by the Jilin Province department of education Science and Technology Plan Projects (Grant NO. 201658 and Grant NO. 201659), partially by the Beihua University Dr. Scientific Research Fund (Grant NO. 2015557). are not suitable for real-time or near real-time processing. Especially in the field of bacterial identification, the image size is different, the shapes are different, the gray scale distribution range is narrow, the local chaotic clusters, the boundary is fuzzy, and the same algorithm can't be used to carry out the key pixel recognition.

Complex network is an important research object in complex system theory. The concepts and methods related to complex network theory are the current research hotspots. The complex network uses the mathematical graph theory to establish the model, only consider the relative position between the nodes and other topological relations, the order of the network nodes, nodes and other places less concerned about the location. The overall rotation and translation of the network graph have no effect on the complex network topology. Therefore, if we can use the complex network as a description model of the plane image boundary shape, and on this basis to establish shape recognition algorithm, then this algorithm will be able to effectively adapt to the image boundary shape changes. In this paper, based on the existing research, the complex network method is applied to the field of image pixe recognition. Aiming at the application environment of bacterial image recognition, a key pixel recognition method based on complex network is proposed. The method has the advantages of simple process and high recognition efficiency. The recognition method has the characteristics of plane rotation invariance, translation invariance, scaling invariance and a certain degree of noise tolerance[1, 2].

\section{SPARSE NETWORK MODEL OF IMAGE}

The description of the image as a complex network model is based on the complex network theory for image recognition of the premise and foundation. The complex network model is divided into unqualified network, undirected weight network, directed network and directed weight network. 
We use the directed and weighted network to describe the image. Super pixel is a region-based image segmentation approach and aims to represent images with a limited number of super pixels which are clusters of neighboring real pixels in an image[3]. The super pixels in the image are taken as the nodes of the network, and the luminance difference $\left|I\left(p_{i}\right)-I\left(p_{j}\right)\right|$ of the two super pixels $[i, j]$ is used as the weight between the two points $w_{i, j}$, when the image is grayscale. When the image is a color map, the edge weight $w_{i, j}$ between node $i$ and $j$ is the RGB European distance of them.

$$
W_{i, j}=\frac{\sqrt{\left(R_{i}-R_{j}\right)^{2}+\left(G_{i}-G_{j}\right)^{2}+\left(B_{i}-B_{j}\right)^{2}}}{\sqrt{3}}
$$

Where R, G, and B are the RGB values of the pixels, respectively. The direction of the edge in the network is the brightness of the pixels point to the brightness of small pixels.

In order to make the uniform network into a complex network, we calculate the Euclidean distance between any two pixels. Then set the radius threshold $r$ and delete the links between the nodes in the network where the distance are greater than $r$. The final weight of the complex network links $w_{i j}$ is given by the formula (2)[4-7]. The complex network model of the image is constructed as shown in Fig. 1. The characteristics of the complex network model of image are shown in the Table 1.

$$
w_{i j}=\left\{\begin{array}{l}
\left|I\left(p_{i}\right)-I\left(p_{j}\right)\right|, \quad\left(\operatorname{dist}\left(p_{i}, p_{j}\right) \leq r\right) \\
0, \quad\left(\operatorname{dist}\left(p_{i}, p_{j}\right)>r\right)
\end{array}\right.
$$

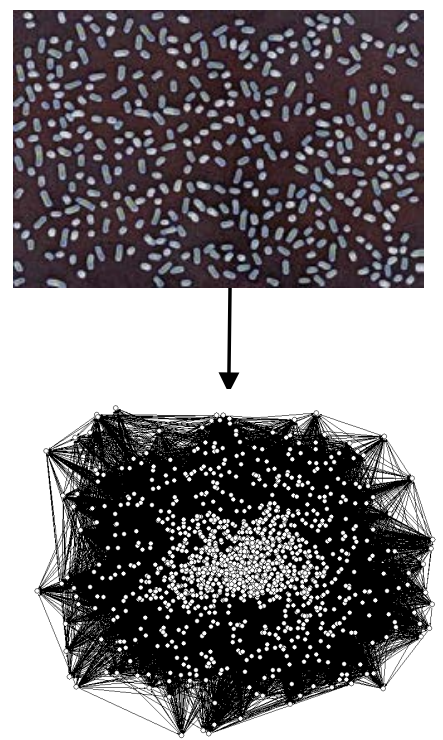

Fig. 1. Complex network model of image. The picture has been done using the software Pajek[8]
TABLE I THE CHARACTERISTICS OF THE COMPLEX NETWORK MODEL

\begin{tabular}{|c|c|}
\hline Characteristic & Value \\
\hline Node & 1332 \\
\hline Link & 63937 \\
\hline $\begin{array}{c}\text { Average in-out- } \\
\text { degree }\end{array}$ & 3723.6 \\
\hline Average in-closeness & 0.083 \\
\hline $\begin{array}{c}\text { Average out- } \\
\text { closeness }\end{array}$ & 0.0862 \\
\hline $\begin{array}{c}\text { Average } \\
\text { betweenness }\end{array}$ & 379.78 \\
\hline $\mathbf{r}$ & 100 \\
\hline
\end{tabular}

\section{INTEREST POINT DETECTION}

Complex network model could reveal the complex structure and function of topological relationship between elements more clearly. There are many different centrality measures of the nodes of a network[9, 10], each one of different nature and with different applications. We propose three different methods, which are degree, clustering coefficient and betweenness centrality, in the analysis of complex networks.

\section{A. The Characterization of the Degree Centrality}

Degree is sometimes called degree in some literature, to emphasize its use as a centrality measure. In directed networks, nodes have both an in-degree and an out-degree, and both may be useful as measures of centrality in the appropriate circumstances. Although degree centrality is a simple centrality measure, it can be very illuminating[10].

The degree of a node in a network is the sum of weight of all the links connected to it. For image network models, there are an out-degree $k_{\text {out }}$ and an in-degree $k_{\text {in }}$ which are, respectively, the sum of regulating and regulated relations at the node, as shown in formula (3).

$$
k_{i}^{\text {out }}=\sum_{j=1}^{n} A_{i j}, \quad k_{j}^{\text {in }}=\sum_{i=1}^{n} A_{i j}
$$

Where $A$ is the adjacency matrix of network, $A_{i j}$ is an element of matrix $A, A_{i j}=1$ if there is a link from node $i$ to node $j$. The total degree $k_{i}$ of a node $n_{i}$ can be computed by the sum of them, i.e., $k_{i}=k_{i}^{\text {in }}+k_{i}^{\text {out }}$. The number of edges $m$ in a directed network is equal to the total number of ingoing ends of edges at all nodes, or equivalently to the total number of outgoing ends of edges, so

$$
m=\sum_{i=1}^{n} k_{j}^{i n}=\sum_{i=1}^{n} k_{j}^{\text {out }}=\sum_{i j} A_{i j}
$$

Thus the mean in-degree cin and the mean out-degree cout of every directed network are equal:

$$
c_{\text {in }}=\frac{1}{n} \sum_{i=1}^{n} k_{i}^{\text {out }}=\frac{1}{n} \sum_{j=1}^{n} k_{j}^{\text {out }}=c_{\text {out }}
$$

The frequency distribution of node degrees is the most fundamental network properties. We defined $P(k)$ as the fraction of nodes in the network with degree $k$. The degree 
distribution of network is a function describing the quantities $P(k)$. The cumulative distribution function $P_{c}(k)$ is the fraction of vertices that has degree $k$ and greater.

\section{B. The Characterization of Betweenness Centrality}

The betweenness centrality of a node $i$ is defined to be the number of the shortest paths that pass through $i$. It express the extent to a node lies on the shortest paths between other nodes. Nodes with high betweenness centrality may have considerable influence within a network by virtue of their control over information passing between others. The nodes with highest betweenness in our message-passing scenario are the ones through which the largest number of messages pass, and if those nodes get to see the messages in question as they pass, or if they get paid for passing the messages along, they could derive a lot of power from their position within the network. The nodes with highest betweenness are also the ones whose removal from the network will most disrupt communications between other nodes because they lie on the largest number of paths taken by messages. Nonetheless, betweenness centrality may still be an approximate guide to the influence nodes have over the flow of information between others.

In a directed network the shortest path between two vertices depends, in general, on the direction you travel in. The shortest path from A to B is different from the shortest path from B to A. Indeed there may be a path in one direction and no path at all in the other. Thus it is important in a directed network explicitly to include the path counts in either direction between each vertex pair. Mathematically, let $n_{j \mathrm{k}}^{i}$ be 1 if node $i$ lies on the shortest path from $j$ to $k$ and 0 if it does not. Then the betweenness $B_{i}$ is given by $B_{i}=\sum_{j \neq k} n_{j k}^{i}$.

\section{The characteristics of the network key nodes}

We measure the characteristics of the network node according to the topology of the complex network, after constructing the complex network model of the image. And then according to the structural characteristics of the node to identify the key nodes of the network, which is the interest point of image. The feature of node $i$ is

$$
\zeta_{i}=\alpha \operatorname{Deg}_{i}+(1-\alpha) \text { Ben }_{i}
$$

where $\operatorname{Deg}_{i}$ is the outdegree of node $i . B e n_{i}$ is the betweenness of node $i$. Degree use as a centrality measure, although degree centrality is a simple centrality measure[10].

\section{RESUlTS AND DisCUSSION}

In order to verify the effectiveness of the proposed algorithm, we use this method for waterborne bacterial images. The bacterial image as shown in Fig. 2. The characteristics of the complex network model of image are shown in the Table 2. Fig.3. shows the results produced by our interest point detection algorithm. This method based on degree and betweenness centrality was applied by filtering the best 5\%$10 \%$ of the points with highest interest. Using this method can be obtained accurately identify the interest points of the vehicle target in the image. Fig. 4 shows the results produced by Harris interest point detection algorithm. Fig. 5 shows the results produced by SIFT interest point detection algorithm.

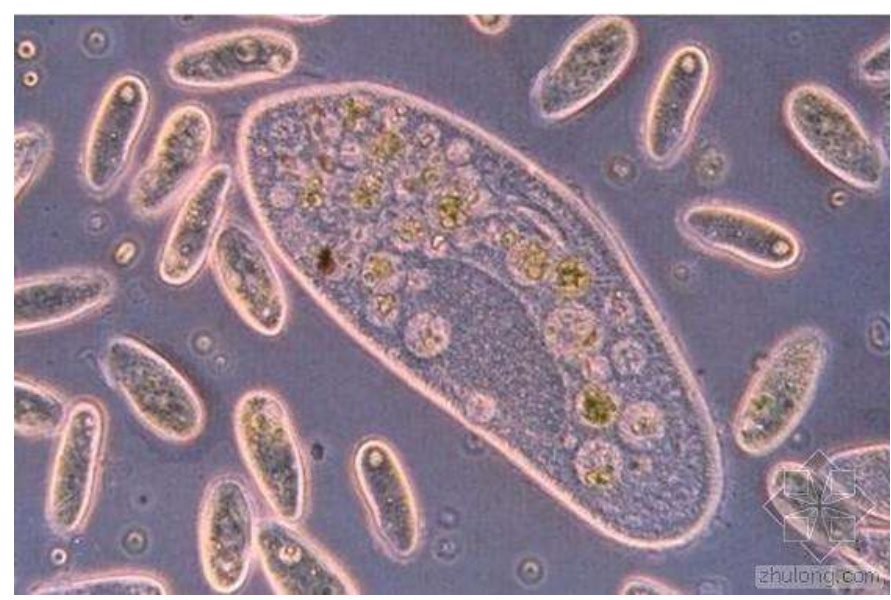

Fig. 2. The interest point identify results of our algorithm

TABLE II. THE CHARACTERISTICS OF THE COMPLEX NETWORK MODEL

\begin{tabular}{|c|c|}
\hline Characteristic & Value \\
\hline Node & 3472 \\
\hline Link & 210514 \\
\hline Average in-out-degree & 4161.9 \\
\hline Average in-closeness & 0.0328 \\
\hline Average out-closeness & 0.049 \\
\hline Average betweenness & 6619.5 \\
\hline $\mathbf{r}$ & 60 \\
\hline
\end{tabular}

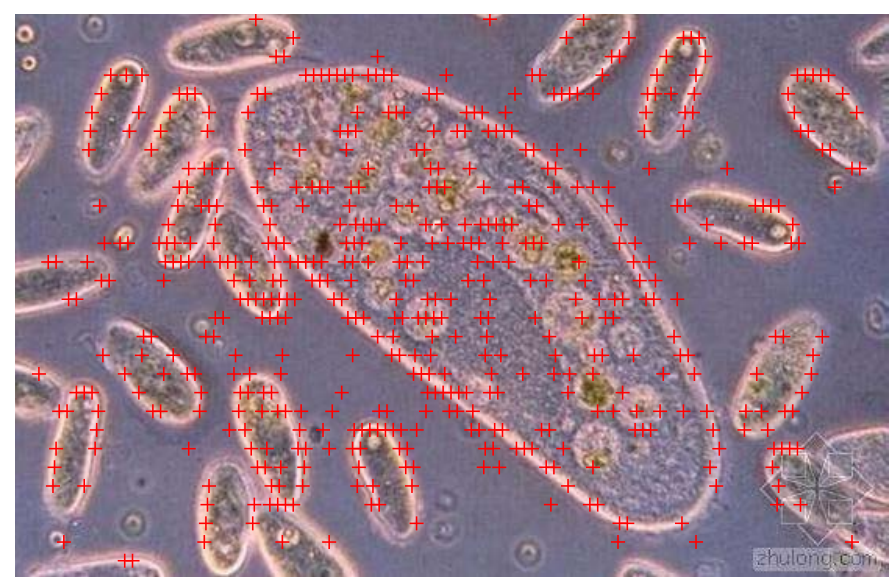

Fig. 3. The interest point identify results of our algorithm 


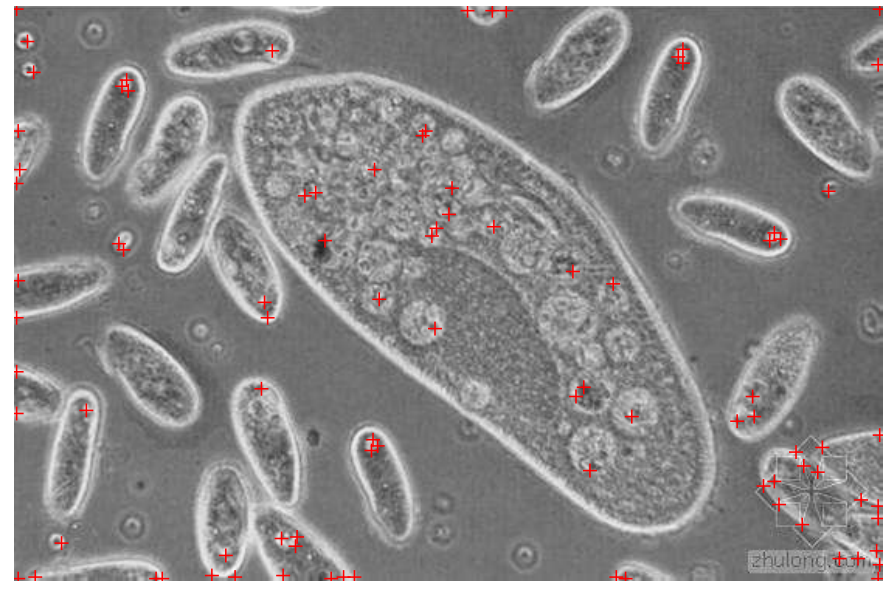

Fig. 4. The interest point identify results of Harris algorithm

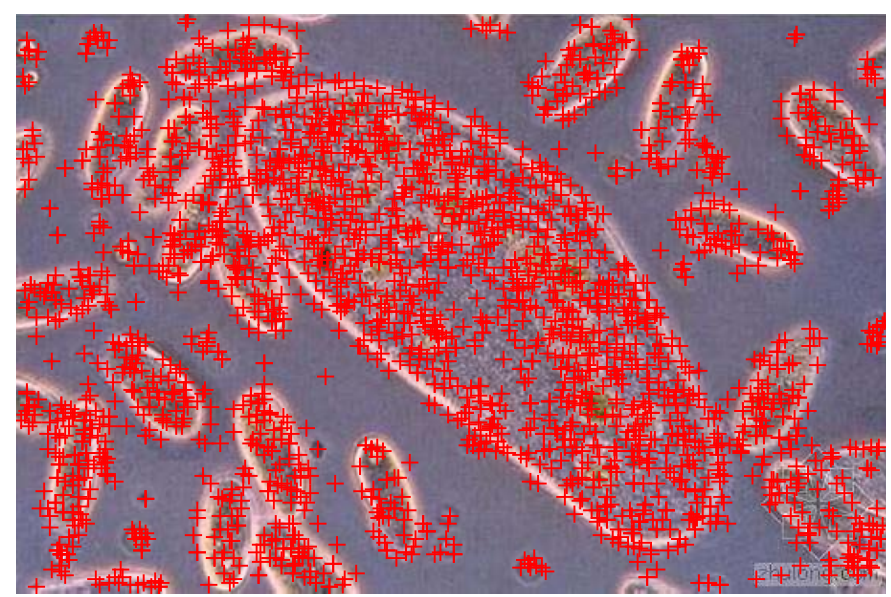

Fig. 5. The interest point identify results of SIFT algorithm

The empirical tests show that the results obtained by three methods in the same picture are quite different. We compare in two different test images the interest point detect results achieved by our complex network based algorithm with Harris and SIFT algorithm. We measured the effectiveness of the results produced by these algorithms using the ratio of the effective nodes to the identified nodes. In a certain sense, our algorithm improves the efficiency and accuracy of image target recognition.

\section{CONCLUSION}

This paper presents a new interest point detection algorithm in waterborne bacterial images based on complex network. Firstly, the complex network model of image is constructed based on the characteristics of image pixels. Then identify the interest nodes according to two topological features of image complex network model, which are degree and betweenness. The experimental results show that our methods are more efficient and accurate for the recognition of interest nodes of image objects compared with the traditional method.

\section{ACKNOWLEDGMENT}

This work is supported mostly by the Key Education Research Project of Beihua university, partially by the Jilin Province Science and Technology Development Project(Grant NO. 20170520057JH), partially by the Jilin Province department of education Science and Technology Plan Projects (Grant NO. 201658 and Grant NO. 201659), partially by the Beihua University Dr. Scientific Research Fund (Grant NO. 2015557).

\section{REFERENCES}

[1] X. Sun, K. Zhao, Z. Lu, T. Nie, and L. Zhou, "Scale-invariant interest point detection in images based on complex network analysis", ICIC Express Letters, Part B: Applications, vol. 7, pp. 525-530, 2016.

[2] J. Tang, Y. Chen, B. Jiang, and B. Luo, "Image Modeling and Feature Extraction Method Based on Complex Network", Computer Engineering, vol. 39, pp. 243-7, 05/ 2013.

[3] O. Cuadros, G. Botelho, F. Rodrigues, and J. B. Neto, "Segmentation of large images with complex networks," in 25th Conference on Graphics, Patterns and Images, SIBGRAPI 2012, August 22, 2012 - August 25, 2012, Ouro Preto, Brazil, 2012, pp. 24-31: IEEE Computer Society.

[4] J. d. A. S. Wesley Nunes Gonçalves, Odemir Martinez Bruno, "A Rotation Invariant Face Recognition Method Based on Complex Network," presented at the 15th Iberoamerican Congress on Pattern Recognition, Sao Paulo, 2010.

[5] A. R. Backes and O. M. Bruno, "Shape classification using complex network and Multi-scale Fractal Dimension", Pattern Recognition Letters, vol. 31, pp. 44-51, 2010.

[6] R. Criado, M. Romance, and A. Sanchez, "Interest point detection in images using complex network analysis", Journal of Computational and Applied Mathematics, vol. 236, pp. 2975-2980, Jun 2012.

[7] R. Criado, M. Romance, and A. Sanchez, "A post-processing method for interest point location in images by using weighted line-graph complex networks", International Journal of Bifurcation and Chaos in Applied Sciences and Engineering, vol. 22, p. 1250163, 2012.

[8] W. d. Nooy, A. Mrvar, and V. Batagelj, Exploratory social network analysis with Pajek (Structural analysis in the social sciences, no. 34). New York: Cambridge University Press, 2011.

[9] M. E. J. Newman, "The structure and function of complex networks", Siam Review, vol. 45, pp. 167-256, Jun 2003, Art. no. Pii s0036144503424804.

[10] M.E.J.Newmen, Networks: An Introduction. New York: Oxford University Press, 2010. 